Nicolas Molnarfi, $\mathrm{PhD}$

Thomas Prod'homme, PhD*

Ulf Schulze-Topphoff, $\mathrm{PhD}$

Collin M. Spencer, BS Martin S. Weber, MD Juan C. Patarroyo, BS Patrice H. Lalive, MD Scott S. Zamvil, MD, $\mathrm{PhD}$

Correspondence to

Dr. Zamvil:

zamvil@ucsf.neuroimmunol.org

\section{Glatiramer acetate treatment negatively regulates type I interferon signaling OPEN}

\section{ABSTRACT}

Objective: Glatiramer acetate (GA; Copaxone), a disease-modifying therapy for multiple sclerosis (MS), promotes development of anti-inflammatory (M2, type II) monocytes that can direct differentiation of regulatory $T$ cells. We investigated the innate immune signaling pathways that participate in GAmediated M2 monocyte polarization.

Methods: Monocytes were isolated from myeloid differentiation primary response gene 88 (MyD88)deficient, Toll-IL-1 receptor domain-containing adaptor inducing interferon (IFN)- $\beta$ (TRIF)-deficient, IFN- $\alpha / \beta$ receptor subunit 1 (IFNAR1)-deficient, and wild-type (WT) mice and human peripheral blood. GA-treated monocytes were stimulated with Toll-like receptor ligands, then evaluated for activation of kinases and transcription factors involved in innate immunity, and secretion of proinflammatory cytokines. GA-treated mice were evaluated for cytokine secretion and susceptibility to experimental autoimmune encephalomyelitis.

Results: GA-mediated inhibition of proinflammatory cytokine production by monocytes occurred independently of MyD88 and nuclear factor-кB, but was blocked by TRIF deficiency. Furthermore, GA did not provide clinical benefit in TRIF-deficient mice. GA inhibited activation of p38 mitogen-activated protein kinase, an upstream regulator of activating transcription factor (ATF)-2, and c-Jun N-terminal kinase 1, which regulates IFN regulatory factor 3 (IRF3). Consequently, nuclear translocation of ATF-2 and IRF3, components of the IFN- $\beta$ enhanceosome, was impaired. Consistent with these observations, GA inhibited production of IFN- $\beta$ in vivo in WT mice, but did not modulate proinflammatory cytokine production by monocytes from IFNAR1-deficient mice.

Conclusion: Our results demonstrate that GA inhibits the type I IFN pathway in M2 polarization of monocytes independently of MyD88, providing an important mechanism connecting innate and adaptive immune modulation in GA therapy and valuable insight regarding its potential use with other MS treatments. Neurol Neuroimmunol Neuroinflamm 2015;2:e179; doi: 10.1212/NXI.0000000000000179

\section{GLOSSARY}

APC $=$ antigen-presenting cells; $\mathbf{B M D M}=$ bone marrow-derived monocytes; $\mathbf{c A M P}_{\mathbf{i}}=$ intracellular cyclic adenosine 3',5'-monophosphate; $\mathbf{E A E}=$ experimental autoimmune encephalomyelitis; $\mathbf{G A}=$ glatiramer acetate; IFN = interferon; IFNAR $=$ type I interferon receptor; IFNAR1 $=$ interferon- $\alpha / \beta$ receptor subunit- 1 ; IL $=$ interleukin; IRF3 $=$ interferon regulatory factor 3; JNK1 = c-Jun N-terminal kinase 1; LPS = lipopolysaccharide; LTA = lipoteichoic acid; $\mathbf{M H C}=$ major histocompatibility complex; $\mathbf{M S}=$ multiple sclerosis; $\mathbf{M y D 8 8}=$ myeloid differentiation primary response gene 88; $\mathbf{N F}=$ nuclear factor; PBS = phosphate-buffered saline; PI3K = phosphoinositide 3-kinase; STAT = signal transducers and activators of transcription; Th $=$ T helper; TLR $=$ Toll-like receptor; TNF $=$ tumor necrosis factor; Treg $=$ regulatory T cells; TRIF $=$ Toll-IL-1 receptor domain-containing adaptor inducing interferon- $\beta$; $\mathbf{W T}=$ wild-type.

Glatiramer acetate (GA; copolymer-1, Copaxone), a random basic copolymer, is prescribed for treatment of relapsing-remitting multiple sclerosis (MS). Previously, it was demonstrated that GA therapy induces anti-inflammatory M2 (type II) myeloid cells. ${ }^{1-3}$ GA-induced M2 monocytes reversed ongoing paralysis in experimental autoimmune encephalomyelitis (EAE) and, as

\footnotetext{
*These authors contributed equally to this work.

From the Department of Neurology and Program in Immunology (N.M., T.P., U.S.-T., C.M.S., J.C.P., S.S.Z.), University of California, San Francisco; the Institute of Neuropathology and Department of Neurology (M.S.W.), University Medical Center, Georg-August University, Göttingen, Germany; the Department of Pathology and Immunology (P.H.L.), Faculty of Medicine, University of Geneva; and the Department of Neurosciences (P.H.L.), Division of Neurology, University Hospital of Geneva, Switzerland. N.M. is currently affiliated with the Department of Pathology and Immunology, Faculty of Medicine, University of Geneva, and Department of Neurosciences, Division of Neurology, University Hospital of Geneva, Switzerland. T.P. is currently affiliated with Momenta Pharmaceuticals, Cambridge, MA. U.S.-T. is currently affiliated with Silence Therapeutics GmbH, Berlin, Germany. J.C.P. is currently affiliated with Vedanta Biosciences, Inc., Cambridge, MA.

Funding information and disclosures are provided at the end of the article. Go to Neurology.org/nn for full disclosure forms. The Article Processing Charge was paid by the authors.

This is an open access article distributed under the terms of the Creative Commons Attribution-NonCommercial-NoDerivatives License 4.0 (CC BY-NC-ND), which permits downloading and sharing the work provided it is properly cited. The work cannot be changed in any way or used commercially.
} 
antigen-presenting cells (APC), promoted expansion of regulatory $\mathrm{T}$ cells (Treg) and $\mathrm{T}$ helper (Th) 2 cells. $^{3}$ Thus, induction of M2 APC is a proximal event responsible for T-cell regulation associated with GA therapy.

While the molecular mechanisms required for GA-mediated M2 differentiation and therapeutic efficacy of GA have not been fully elucidated, it is recognized that GA inhibits monocyte reactivity in response to engagement of Toll-like receptors (TLRs), ${ }^{1,3}$ suggesting that modulation of innate signaling could represent a principal mechanism of action of GA. In general, TLR engagement triggers activation through interaction with adaptor molecule myeloid differentiation primary response gene 88 (MyD88) or Toll-IL-1 receptor domain-containing adaptor inducing interferon (IFN)- $\beta$ (TRIF). Both pathways are important in CNS autoimmunity. ${ }^{4-6}$ MyD88-dependent signaling is associated with activation of the nuclear factor $(\mathrm{NF})-\kappa \mathrm{B}$ pathway and is required for Th17 differentiation in EAE. ${ }^{7}$ In contrast, signaling via TRIF leads to activation of IFN regulatory factor 3 (IRF3) transcription factor and subsequent production of IFN- $\beta .^{8}$ Signaling through TRIF, IRF3, or the type I IFN receptor (IFNAR) also influences development of Th17 cells and EAE.,6,9 Thus, reduction of proinflammatory cytokines observed following stimulation of M2 monocytes could indicate that GA modulates MyD88, TRIF, or both pathways.

The goal of this investigation was to identify the molecular pathways participating in differentiation of M2 APC by GA. Our results, which demonstrate that GA downregulates type I IFN signaling, provide a key mechanism responsible for inhibition of proinflammatory APC polarization by GA treatment.

METHODS Mice. Female 8- to 10-week-old C57BL/6 wildtype (WT), IFN- $\alpha / \beta$ receptor subunit-1 (IFNAR1)-deficient, TRIF-deficient, and CD11b-deficient mice were obtained from Jackson Laboratories (Bar Harbor, ME). C57BL/6 MyD88deficient mice were provided by Dr. A. DeFranco (University of California, San Francisco). Mice were maintained and handled in accordance with UCSF Institutional Animal Care and Use Committee regulations for the humane use of animals in research.

EAE induction. C57BL/6J mice were injected SC with $50 \mu \mathrm{g}$ MOG p35-55 (Auspep, Parkville, Australia) emulsified in complete Freund's adjuvant containing $200 \mu \mathrm{g}$ heat-killed Mycobacterium tuberculosis on day 0. Mice received $200 \mathrm{ng}$ Bordetella pertussis toxin (List Biological Laboratories, Campbell, CA) IV in $0.2 \mathrm{~mL}$ phosphate-buffered saline (PBS) on days 0 and 2. Mice were observed daily. Mice received one SC injection of a $0.1-\mathrm{mL}$ emulsion consisting of $250 \mu \mathrm{g}$ GA (provided by Teva Pharmaceutical Industries, Petah Tikva, Israel) in an equal volume of PBS and incomplete Freund's adjuvant in the upper flanks on the same day of immunization (day 0). Clinical scores were assessed as follows: $0=$ no clinical disease, $1=$ loss of tail tone only, $2=$ mild monoparesis or paraparesis, $3=$ severe paraparesis, $4=$ paraplegia or quadriparesis, and $5=$ moribund or death.

Monocyte isolation and culture. $\mathrm{CD} 11 \mathrm{~b}^{+} \mathrm{CD} 11 \mathrm{c}^{-}$macrophages (purity $>98 \%$ ) were obtained from C57BL/6 WT, MyD88-deficient, IFNAR1-deficient, TRIF-deficient, and CD11b-deficient mice. Bone marrow-derived monocytes (BMDM) were obtained following in vitro GA or vehicle (PBS) treatment, for 7 days in RPMI, 10\% FCS, and 10\% L929 cell-conditioned medium (L929-CM), which was used as a source of murine M-CSF. Mouse BMDM and peripheral macrophages stained positively for $\mathrm{CD} 11 \mathrm{~b}, \mathrm{~F} 4 / 80$, and major histocompatibility complex (MHC) class II (I-A) cell surface molecules. For cyclic adenosine 3',5'-monophosphate (cAMP) quantification, DNA binding, and ELISA assays, monocytes were cultured in 96-well plates at $2.5 \times 10^{4}$ cells/well in 200 $\mu \mathrm{L}$ culture media. For Western blot analyses, monocytes differentiated in the presence or absence of GA were resuspended at $10 \times 10^{6}$ cells $/ \mathrm{mL}$ in culture media, and $2 \mathrm{~mL}$ were plated in 6 -well plates at $37^{\circ} \mathrm{C}$ for 1 hour, then activated with indicated stimuli. Human peripheral blood buffy coat monocytes were isolated from healthy volunteers as previously described. ${ }^{10}$ Human monocytes expressed cell surface CD14, MHC class II (HLA-DR), and CD68 molecules. Monocytes were cultured in 96-well plates at $5 \times 10^{4}$ cells/well in $200 \mu \mathrm{L}$ media, and activated with the indicated stimuli for 24 hours. Monocytes were stimulated with forskolin (an agonist of adenylyl cyclase and serves as a positive control for induction of intracellular cAMP), lipopolysaccharide (LPS), Poly(I:C), lipoteichoic acid (LTA), and Pam3CSK4, which were purchased from InvivoGen (San Diego, CA).

ELISA. Production of tumor necrosis factor (TNF), interleukin (IL) -6 , and IFN- $\beta$ was measured in serum or culture supernatants by ELISA (eBioscience, San Diego, CA; R\&D Systems, Minneapolis, $\mathrm{MN}$ ), per manufacturer protocol. Intracellular cAMP $\left(\mathrm{cAMP}_{\mathrm{i}}\right.$ ) was detected using Biotrack kit (GE Healthcare, Cleveland, $\mathrm{OH})$.

DNA binding. DNA binding of ATF-2 and IRF3 was quantitated from monocyte nuclear extracts using the ELISAbased Transfactor (Clontech, Mountain View, CA) and TransAM (Active Motif, Carlsbad, CA) kits, respectively.

Western blot. Total cell lysates were prepared from monocyte cultures and subjected to Western blot analysis as described previously. ${ }^{3}$ Nitrocellulose membranes were probed $\left(4^{\circ} \mathrm{C}\right.$, overnight) with antibodies against phospho-Akt1 ( $\left.\operatorname{Ser}_{473}\right)$, phospho-JNK $\left(\mathrm{Thr}_{183} /\right.$ Tyr $\left.{ }_{185}\right)$, phospho-p38 MAP kinase $\left(\mathrm{Thr}_{180} / \mathrm{Tyr}_{182}\right)$, phosphoIкB $\alpha \quad\left(\mathrm{Ser}_{32 / 36}\right), \quad$ glyceraldehyde-3-phosphate dehydrogenase $(\mathrm{GAPDH})$, total STAT1, and total Akt1/2/3 (Cell Signaling Technology, Danvers, MA). Horseradish peroxidase-conjugated goat anti-mouse (Thermo Scientific, Waltham, MA) or sheep anti-rabbit (Covalab, Villeurbanne, France) antibodies were used for secondary detection. Antibody-bound proteins were detected 
by the Uptilight HRP Blot Chemiluminescent substrate (GE Healthcare). Western blots were digitized and quantified using NIH imageJ software (version 1.44).

Mouse phospho-Akt (Ser $\left.{ }_{473}\right)$ cell-based ELISA. PathScan Phospho-Akt1 ( Ser $\left._{473}\right)$ Sandwich ELISA Kit (Cell Signaling Technology) was used to detect endogenous phospho-Akt1 $\left(\operatorname{Ser}_{473}\right)$ protein. Equal concentrations of protein extract $(100$ $\mu \mathrm{g} / \mathrm{mL}$ ) were analyzed for each sample. The magnitude of absorbance $(450 \mathrm{~nm})$ was proportional to the quantity of phospho-Akt1 $\left(\operatorname{Ser}_{473}\right)$ protein. Values were expressed as a fraction of the amount of phospho-Akt1 ( $\left.\operatorname{Ser}_{473}\right)$ observed in BMDM extract, which was normalized to 1 .

Statistics. Data are presented as the mean \pm SD. For ELISA data, $\mathrm{n}=3$ separate samples for each condition were examined. When required, statistical significance of differences between groups was evaluated using Student paired $t$ test. Differences were considered significant at a value of $p<0.05$. For clinical scores, significance between groups was examined using the MannWhitney $U$ test. A value of $p<0.05$ was considered significant.

RESULTS GA induces activation of PI3K, but not cAMP. Whether GA selectively engages specific receptors is unclear. cAMP and phosphoinositide 3-kinase (PI3K) are second messenger systems that can be activated after receptor engagement, and have been associated with early signaling events in antiinflammatory responses. ${ }^{11}$ As shown in figure 1A, incubation of monocytes with GA did not induce intracellular cAMP levels, as compared to stimulation by the positive control, adenylylcyclase activator forskolin, suggesting that GA-induced M2 differentiation does not involve cAMP. Phosphorylation of Akt on serine 473 is a surrogate marker of PI3K activation. Within 15 minutes, GA induced phosphorylation of Akt as measured both by Western blot (figure 1B) and ELISA (figure 1C). These results suggest that GA activates at least one intracellular second messenger signaling pathway associated with reduction of inflammation in innate immune cells.

GA inhibits TRIF-dependent, but not MyD88-dependent, signaling. MyD88 and TRIF represent the 2 essential adapters of innate immune signaling. Previous data demonstrated that GA treatment was associated with reduction of proinflammatory cytokine production and STAT1 phosphorylation in monocytes following stimulation with LPS, a TLR4 agonist. $^{3}$ All TLRs utilize MyD88 except TLR3, which depends exclusively on TRIF. ${ }^{8}$ TLR 4 signals via both MyD88 and TRIF. Therefore, it was possible that GA could interfere with MyD88 or TRIF. We employed distinct TLR agonists in order to distinguish between these 2 possibilities. First, we observed that GA itself did not alter baseline or ligand-induced expression of TLR2, TLR3, or TLR4 (figure e-1 at Neurology.org/nn), indicating that GA did not influence the capacity to sense TLR agonists. As shown in figure $2 \mathrm{~A}$ and figure $\mathrm{e}-2$, activation of GA-treated monocytes by the
Figure 1 Glatiramer acetate induces timedependent phosphorylation of Akt in monocytes

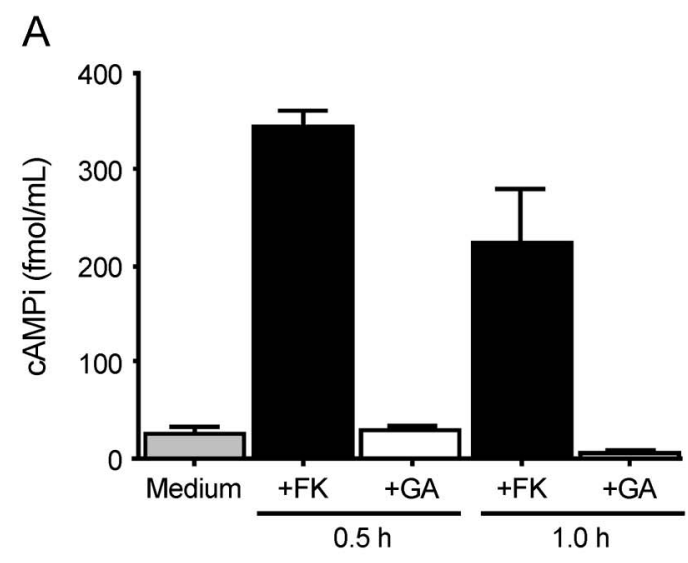

B

Relative fold increase
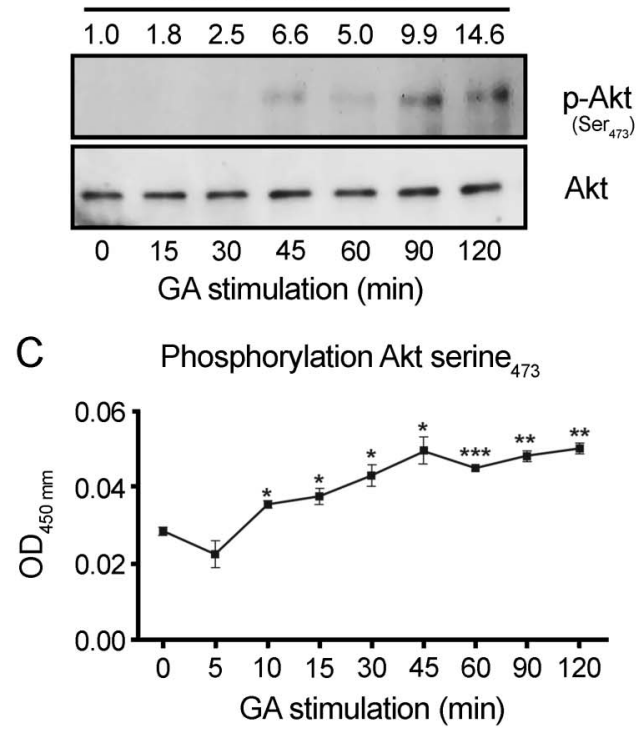

(A) Monocytes were stimulated for 30 or 60 minutes with $50 \mu \mathrm{g} / \mathrm{mL}$ glatiramer acetate (GA) or forskolin (FK), a labdane diterpene, which is an agonist of the enzyme adenylyl cyclase, and serves as a positive control for induction of intracellular cyclic adenosine $3^{\prime}, 5^{\prime}$-monophosphate $\left(\mathrm{cAMP} \mathrm{P}_{\mathrm{i}}\right.$ ). Monocytes were lysed and $\mathrm{cAMP}_{\mathrm{i}}$ was determined by enzyme immunoassay. Values are expressed as the mean $\pm S D(n=3)$. Data are representative of 3 independent experiments. (B, C) Monocytes were stimulated with $50 \mu \mathrm{g} / \mathrm{mL}$ GA for the indicated time periods prior to cell lysis. (B) Cell lysates were then subjected to sodium dodecyl sulfate polyacrylamide gel electrophoresis and immunoblotted with phospho-specific antibodies to serine-473 of Akt1 (phospho-Akt1 [Ser 473 ]). The same blot was stripped and probed for total Akt. Relative ratio represents the fold induction of Akt1 (Ser ${ }_{473}$ ) phosphorylation by GA treatment. (C) Endogenous levels of phospho-Akt1 ( Ser $_{473}$ ) protein were detected by a solid phase sandwich ELISA. The magnitude of absorbance is proportional to the quantity of phospho-Akt1 ( $\mathrm{Ser}_{473}$ ) protein. ${ }^{*} p<0.05, * * p<0.01, * * * p<0.001$ as determined by Student $t$ test. Results shown are representative of 2 separate experiments. 
A
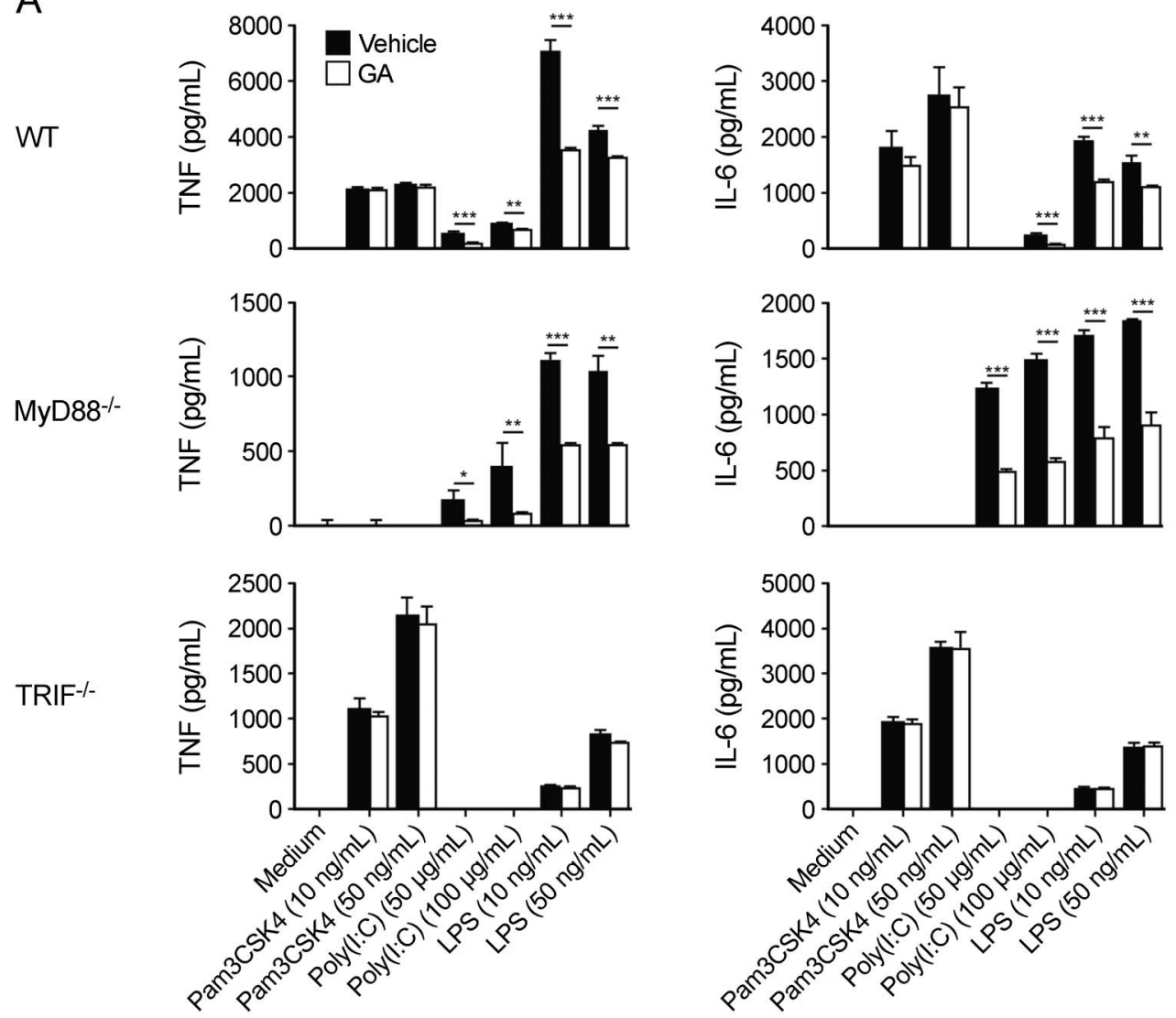

$B$

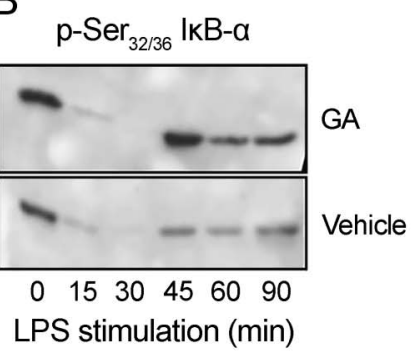

C

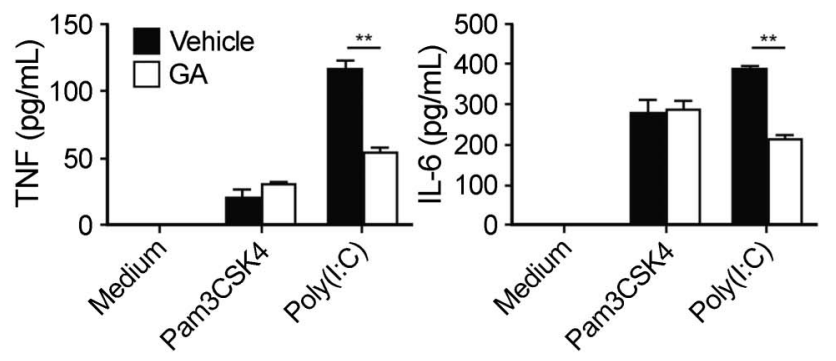

(A) As described previously, ${ }^{3} \mathrm{M} 2$ monocytes were treated in the presence or absence of glatiramer acetate (GA) for 6 days. They were then stimulated with lipopolysaccharide (LPS), Poly(I:C), or Pam3CSK4 for 24 hours. (B) Wild-type (WT) monocytes cultured in the presence or absence of GA were stimulated with LPS $(100 \mathrm{ng} / \mathrm{mL})$ for the indicated duration. Cell lysate proteins were separated by sodium dodecyl sulfate polyacrylamide gel electrophoresis and membranes were probed for phosphorylated $I_{\kappa} B \alpha\left(\operatorname{Ser}_{32 / 36}\right)$. Data are representative of 2 separate experiments. (C) Human peripheral blood monocytes were preincubated for 1 hour with or without $50 \mu \mathrm{g} / \mathrm{mL}$ GA and then cultured for 24 hours in the presence or absence of Poly(l:C) $(10 \mu \mathrm{g} / \mathrm{mL})$ or Pam3CSK4 (100 ng/mL). Tumor necrosis factor (TNF) (left panels) and interleukin (IL)-6 (right panels) secretion was quantitated in cell supernatants by ELISA. Results are presented as mean $\pm S D(n=3) ; * p<0.01$, ${ }^{* * *} p<0.001$ by Student $t$ test. Data presented are representative of 3 independent experiments. MyD88 $=$ myeloid differentiation primary response gene $88 ;$ TRIF $=$ Toll-IL-1 receptor domain-containing adaptor inducing interferon- $\beta$.

TLR2 ligands, Pam3CSK4 or LTA, did not alter TNF and IL-6 secretion. In contrast, secretion of these proinflammatory cytokines was reduced when GA-treated monocytes were activated by the TLR3 ligand, Poly(I:C), or the TLR4 ligand, LPS. These results therefore suggested that GA inhibited TRIFdependent signaling and was independent of MyD88. Similar to monocytes from WT mice, monocytes from MyD88-deficient mice exhibited reduced IL-6 and TNF levels following GA treatment (figure 2A). Engagement of MyD88 activates NF- $\mathrm{B}$. As shown in figure $2 \mathrm{~B}, \mathrm{GA}$ exposure did not impair phosphorylation and degradation of the inhibitor protein ІкB $\alpha$ following LPS treatment. GA exposure also did not significantly alter DNA binding of $\mathrm{p} 50$ or $\mathrm{p} 65 \mathrm{NF}-\mathrm{kB}$ subunits 
(data not shown). These results suggest that the NF- $\mathrm{KB}$ pathway is dispensable for the immunologic effects of GA on monocytes. Further, GA did not reduce proinflammatory cytokine secretion in TRIF-deficient monocytes (figure $2 \mathrm{~A}$ ), providing further support that inhibition of TRIF-dependent signaling is required for GA-mediated reduction of proinflammatory cytokine secretion. As GA treatment of murine monocytes suppressed proinflammatory cytokine production by Poly(I:C), but not Pam3CSK4, we tested how GAtreated human monocytes responded to these 2 TLR ligands. GA treatment of human peripheral blood monocytes decreased TNF and IL-6 secretion induced by Poly(I:C), but not Pam3CSK4 (figure 2C). Thus, as with murine monocytes, these results indicate that GA inhibits proinflammatory cytokine production by human monocytes by blocking TRIF-mediated signaling.

The in vivo contribution of TRIF-dependent signaling in modulating proinflammatory cytokine secretion was also examined during GA treatment. GA-treated WT mice that were stimulated with LPS (figure 3A), but not with Pam3CSK4 (figure $3 \mathrm{~B}$ ), exhibited a significant reduction of serum TNF and IL-6 levels. However, this modulation of TNF and IL- 6 was not observed in GA-treated TRIFdeficient mice following LPS injection (figure 3B). As MyD88-deficient mice are unresponsive to Pam3CSK 4 or LPS, ${ }^{12}$ these mice were evaluated by stimulation with Poly(I:C). GA treatment reduced proinflammatory cytokine serum levels in MyD88deficient mice in response to Poly(I:C) (figure 3C). Thus, these in vivo results further establish that GA inhibits TRIF-dependent, but not MyD88dependent, signaling.

We then addressed whether TRIF deficiency might alter the clinical effect of GA treatment in EAE. TRIFdeficient, but not MyD88-deficient, mice are susceptible to EAE. ${ }^{5-7}$ Clinical severity of EAE in untreated WT and TRIF-deficient mice was similar (figure 3D). However, GA suppressed EAE development in WT mice but not in TRIF-deficient mice. Specifically, the incidence, day of onset, and EAE severity were not altered by GA treatment of TRIF-deficient mice. These in vivo results further confirmed the importance of modulating TRIF-dependent signaling in the clinical activity of GA.

GA treatment inhibits IFN- $\beta$ production by targeting regulatory elements of the IFN- $\beta$ promoter. IFN- $\beta$ is a key cytokine induced via the TRIF-dependent pathway that contributes to both innate and adaptive immune responses. $^{4-6}$ Therefore, we analyzed expression of IFN- $\beta$ in monocytes treated with GA. As anticipated, we observed a reduction of IFN- $\beta$ secretion by GA-treated WT monocytes (figure 4A). Serum IFN- $\beta$ was also decreased in GA-treated mice that were subsequently challenged with LPS (figure 4B).

Through binding to IFNAR and activating the signal transducers and activators of transcription (STAT) signaling pathway, IFN- $\beta$ acts in a feedback manner to amplify monocyte responses to innate stimulation. ${ }^{13}$ Previously, we reported that STAT1 phosphorylation was impaired in M2 monocytes. ${ }^{3}$ Thus, we addressed the importance of IFNAR1 in GA treatment. As shown in figure 4C, IFNAR1 deficiency prevented GA-mediated modulation of IL-6 and TNF secretion. Together, these results suggest that reduction of type I IFN signaling may be a primary effect of GA action on monocytes.

Type I IFN expression requires the coordinated activation of the transcription factors NF- $\mathrm{B}$, IRF3, and ATF-2/c-Jun (AP-1), which comprise the IFN- $\beta$ enhanceosome. ${ }^{14}$ We evaluated the influence of GA treatment on activation of these signaling molecules and their regulators. As described above, GA treatment did not influence NF- $\mathrm{B}$ activation. In contrast, we observed that GA suppressed nuclear translocation of IRF3 in monocytes following stimulation with either LPS or Poly(I:C) (figure 4D). Activation and nuclear translocation of IRF3 are dependent upon phosphorylation by c-Jun N-terminal kinase 1 (JNK1). ${ }^{15}$ In this regard, phosphorylation of JNK1 was reduced in GA-treated monocytes in response to LPS stimulation (figure 4E). Similarly, decreased DNA binding of ATF-2 was observed following incubation with GA (figure 4F and figure e-3), which corresponded to inhibition of p38 MAPK phosphorylation (figure 4G), the major upstream regulator of ATF-2. ${ }^{16}$ Taken together, these results demonstrate that GA treatment inhibited IFN- $\beta$ production by interfering with activation of transcription factors participating in formation of the IFN- $\beta$ enhanceosome.

DISCUSSION Cellular immune responses that result from GA treatment of MS and EAE have been welldescribed. ${ }^{17,18}$ In 2007, it was shown that it is the influence of GA on APCs that is responsible for the amplification of Th2 and Treg cells, a study that provided the first demonstration that M2 monocytes could reverse CNS autoimmune disease and promote T-cell immune modulation in vivo. ${ }^{3}$ At that time the longheld belief that GA-specific $\mathrm{T}$ cells required crossreactivity with myelin self-antigen for therapeutic benefit was disproved. It was also observed that GA treatment was associated with inhibition of STAT1 in monocytes, indicating that GA affected at least one proinflammatory signaling pathway within these cells. ${ }^{3}$ TRIF and MyD88 represent 2 major innate pathways involved in the induction of several proinflammatory responses, including cytokine secretion. Our results 
Figure 3 TRIF deficiency abrogates the immunomodulatory effects of glatiramer acetate treatment on cytokines and experimental autoimmune encephalomyelitis

A

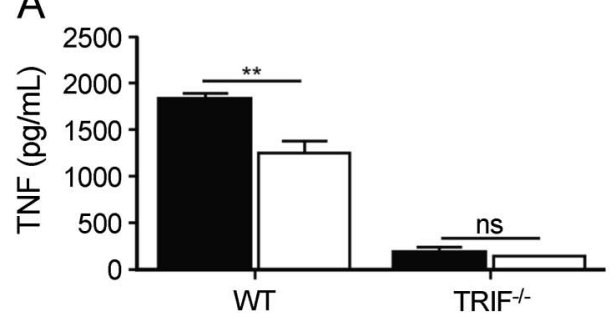

In vivo LPS stimulation
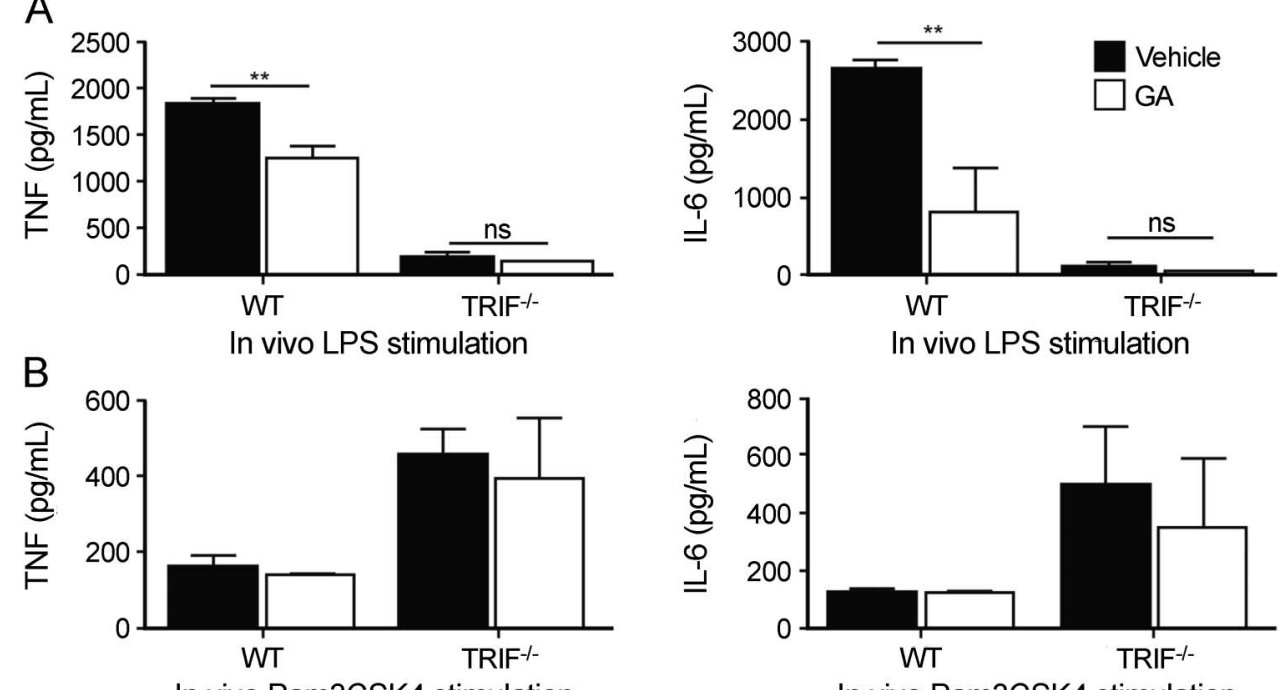

In vivo Pam3CSK4 stimulation
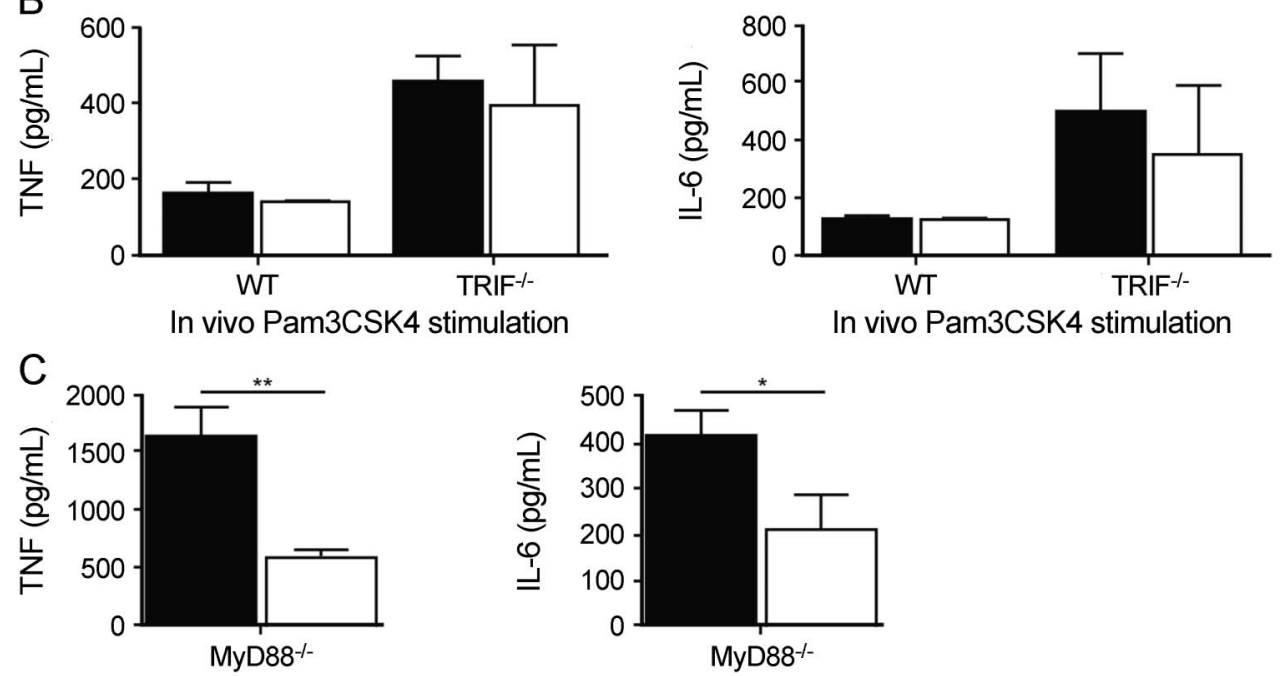

In vivo Poly(l:C) stimulation

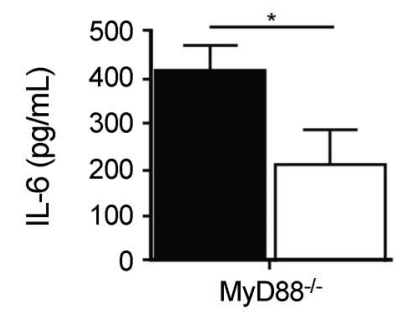

In vivo Poly(l:C) stimulation
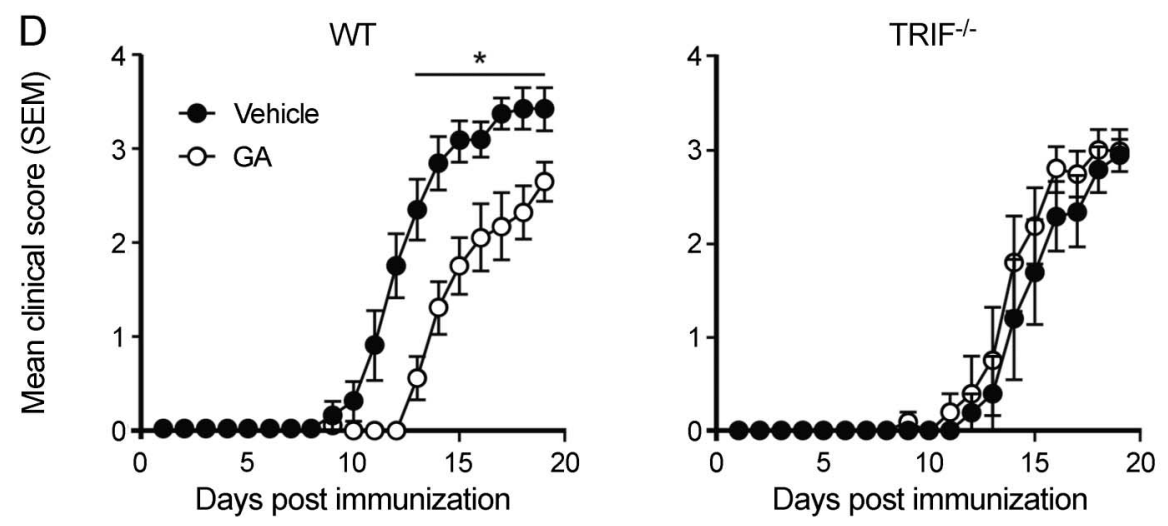

Wild-type (WT) and Toll-IL-1 receptor domain-containing adaptor inducing interferon- $\beta$ (TRIF)-deficient mice treated with glatiramer acetate (GA) or vehicle ( $\mathrm{n}=5 \mathrm{mice} / \mathrm{group}$ ) were injected IP with (A) lipopolysaccharide (LPS) (100 ng/kg) or (B) Pam3CSK4 (100 ng/kg). (C) Myeloid differentiation primary response gene 88 (MyD88)-deficient mice were injected IP with Poly(I:C) $(10 \mu \mathrm{g} / \mathrm{kg})$. Serum levels of tumor necrosis factor (TNF) and interleukin (IL)- 6 were quantitated by ELISA 5 hours after injection. Results are presented as the mean \pm SEM $(n=3)$ of 2 experiments that provided similar results; * $p<0.05$, $* * p<0.01, * * * p<0.001$ as determined by Student $t$ test. (D) On day 0, C57BL/6J WT (left) or TRIF-deficient mice (right) mice were immunized with MOG peptide $(\mathrm{p} 35-55 ; 50 \mu \mathrm{g})$. GA $(250 \mu \mathrm{g})$ was administered once (SC in incomplete Freund's adjuvant [IFA]) on the same day as immunization (day 0). Control mice received a single SC injection of IFA. For all experimental autoimmune encephalomyelitis experiments, mean disease score \pm SEM is shown. $* p<0.05$ as determined by Mann-Whitney $U$ test. Results shown are representative of 3 independent experiments.

demonstrate that GA inhibits the TRIF-dependent pathway, resulting in a reduction in activity of downstream signaling molecules that lead to production of IFN- $\beta$ (figure 5). This immune modulation occurred independently of MyD88 and did not influence NF- $\mathrm{kB}$ activation. These findings provide a key anti-inflammatory mechanism connecting innate and adaptive immune modulation in GA therapy.

Engagement of some cell surface receptors has been associated with anti-inflammatory polarization of myeloid cells. Vasoactive intestinal peptide, which, similar to GA, also exerts anti-inflammatory effects, ${ }^{19}$ 
Figure 4 Glatiramer acetate treatment negatively regulates IFN- $\beta$ production by targeting components of the IFN- $\beta$ enhanceosome

A

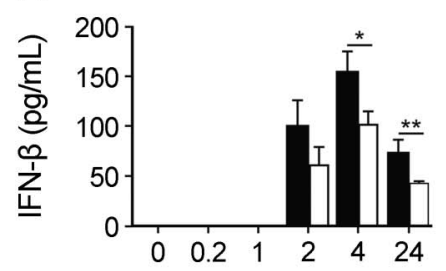

Time after LPS stimulation (h)

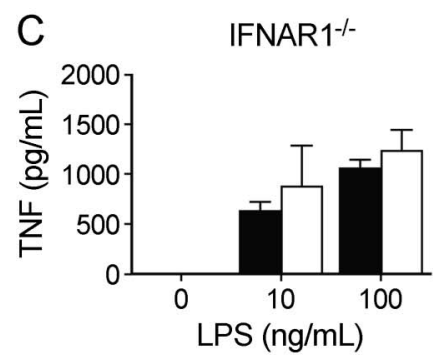

D IRF3 DNA binding

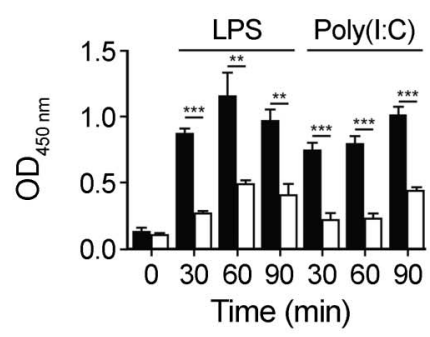

$\mathrm{F}$

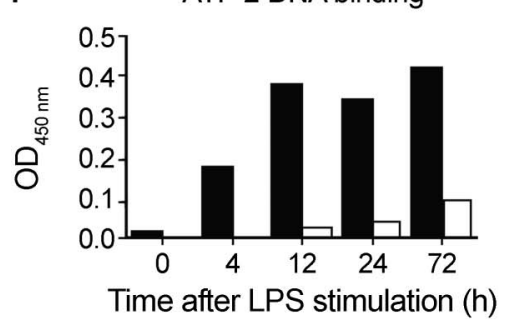

B

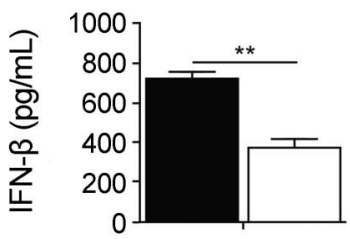

In vivo LPS stimulation

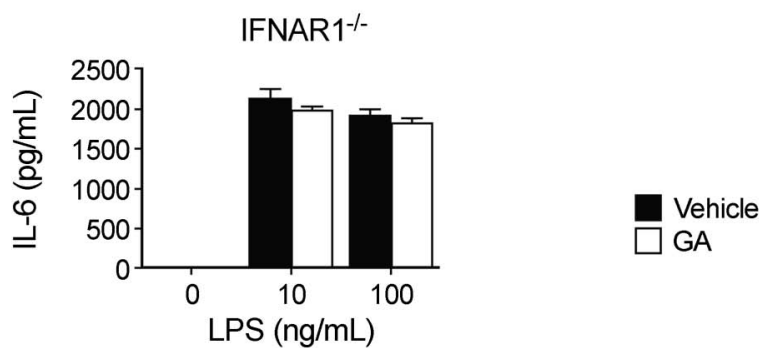

E

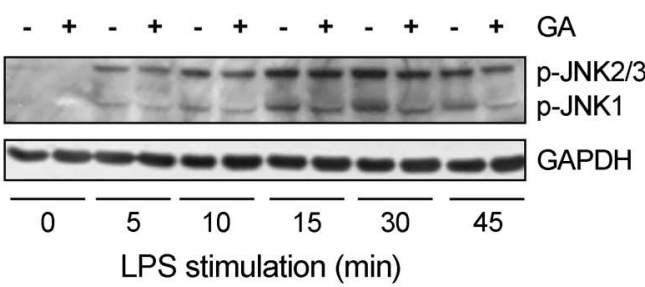

G

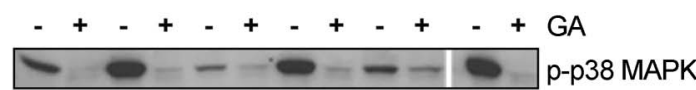

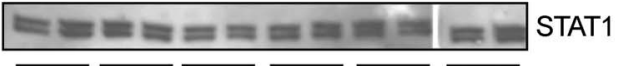

LPS stimulation (min)

(A) Wild-type (WT) monocytes differentiated in the presence or absence of glatiramer acetate (GA) were stimulated with lipopolysaccharide (LPS) (100 ng/mL) for 24 hours. Interferon (IFN)- $\beta$ secretion was quantitated in cell culture supernatants by ELISA. Results are representative of 2 experiments. Data are presented as mean \pm SEM; $* p<0.05, * * p<0.01$ as determined by Student $t$ test. (B) WT mice ( $n=3 /$ group) treated with GA or vehicle were injected IP with LPS (100 ng/kg). Serum levels of IFN- $\beta$ were quantitated by ELISA, 5 hours following injection. Data are representative of 2 separate experiments. (C) Interferon- $\alpha / \beta$ receptor subunit-1 (IFNAR1)-deficient monocytes differentiated in the presence or absence of GA were stimulated with LPS at the indicated dose for 24 hours. Tumor necrosis factor (TNF) and interleukin-6 secretion was quantitated in cell supernatants by ELISA. Results are representative of 3 independent experiments ( $n=3 / g r o u p)$. (D) Monocytes generated in the presence or absence of GA were stimulated with LPS (100 ng/mL) or Poly(l:C) (10 $\mu \mathrm{g} / \mathrm{mL}) \mathrm{for}$ the indicated duration. IRF3 binding activity in nuclear extracts was measured with TransAM IRF3. (E) Monocytes generated in the presence or absence of GA were stimulated with LPS $(100 \mathrm{ng} / \mathrm{mL})$ for the indicated duration. Cell lysate proteins were separated by sodium dodecyl sulfate polyacrylamide gel electrophoresis (SDS-PAGE) and membranes were probed for phosphorylated SAPK/JNK ( $\mathrm{Thr}_{183} / \mathrm{Tyr}_{185}$ ) and total GAPDH. Data are representative of 3 separate experiments. (F) Monocytes differentiated in the presence or absence of GA and stimulated with $100 \mathrm{ng} / \mathrm{mL}$ LPS for the indicated duration. DNA binding of ATF-2 was quantitated from nuclear extracts. (G) Monocytes generated in the presence or absence of GA were stimulated with LPS (100 ng/mL) for the indicated duration, and whole-cell lysates were subjected to SDS-PAGE and Western blot analysis for phosphorylated p38 MAPK ( $\left.\mathrm{Thr}_{180} / \mathrm{Tyr}_{182}\right)$ and total STAT1. Data are representative of 3 separate experiments. 


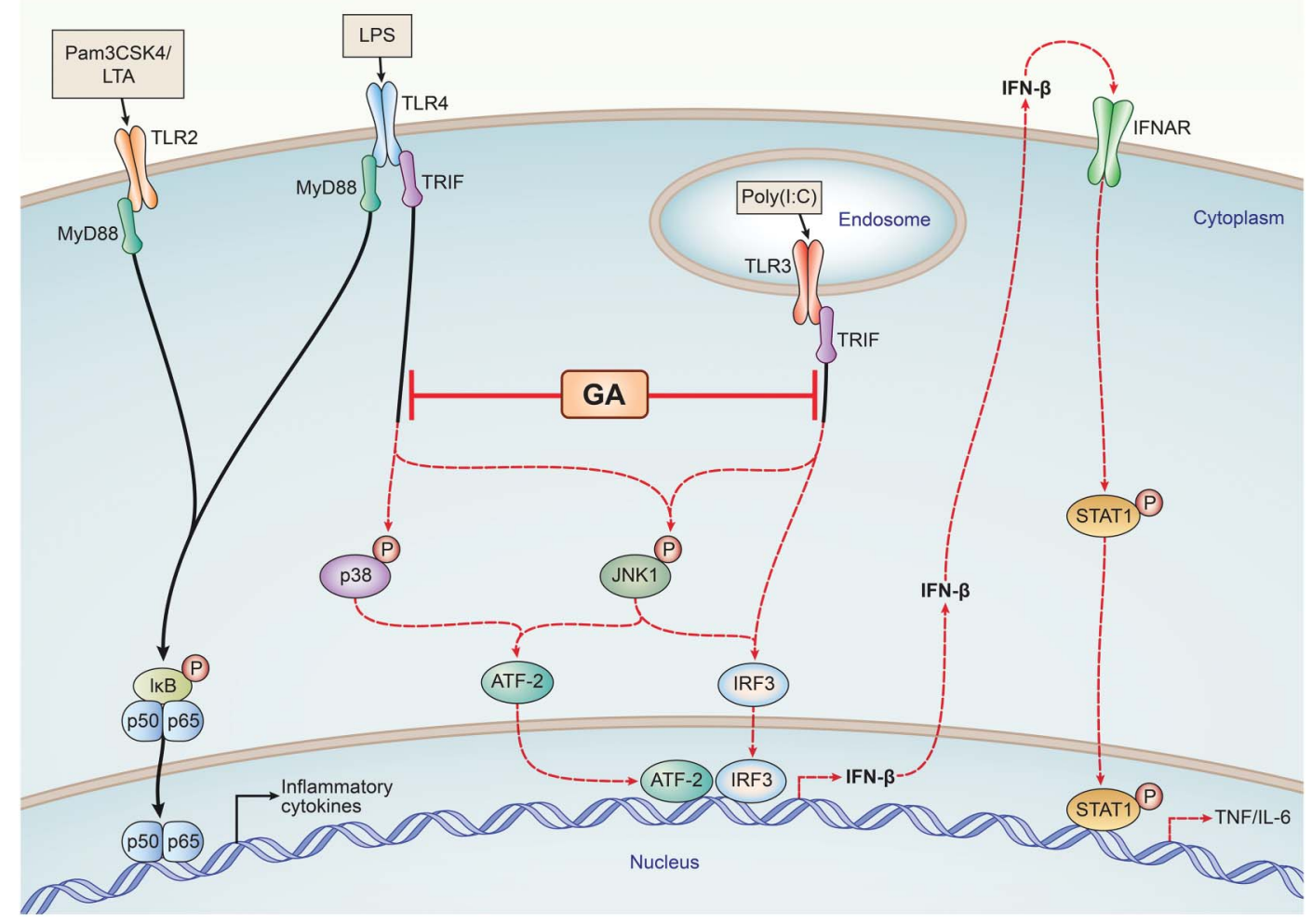

Myeloid differentiation primary response gene 88 (MyD88) and Toll-IL-1 receptor domain-containing adaptor inducing interferon- $\beta$ (TRIF) are 2 major mediators of Toll-like receptor (TLR) signaling that cooperate in promoting innate immune responses. Engagement of TLR2 by Pam3CSK4 or lipoteichoic acid (LTA) initiates the MyD88-dependent pathway resulting in the transcription of proinflammatory cytokines via the induction (black lines) of nuclear factor (NF)- $\mathrm{\kappa B}$. Stimulation of TLR3 by Poly(I:C) induces the recruitment of TRIF, which in turn triggers expression of interferon (IFN)- $\beta$ by activating interferon regulatory factor 3 (IRF3), a substrate of c-Jun $\mathrm{N}$-terminal kinase 1 (JNK1). ${ }^{15}$ Engagement of TLR4 by lipopolysaccharide (LPS) triggers both MyD88-dependent and TRIF-dependent signaling. Both TLR3 and TLR4, but not TLR2, initiate an IFN- $\beta$-positive feedback loop that amplifies the initial response through the IFNAR-signal transducers and activators of transcription 1 (STAT1) signaling axis. The differentiation of M2 monocytes occurred independently of MyD88 and phosphorylation and degradation of the NF-кB inhibitor, IкB $\alpha$. In contrast, TRIF-dependent TLR signaling was negatively regulated in M2 monocytes. Phosphorylation of JNK1 and p38 MAPK and subsequent nuclear translocation (DNA binding) of IRF3 and ATF-2, 2 components of the IFN- $\beta$ enhanceosome, were inhibited. GAmediated M2 monocyte differentiation is associated with inhibition of STAT1 phosphorylation. ${ }^{3}$ Copyright Xavier Studio, reprinted with permission.

triggers the second messengers PI3K and cAMP following receptor engagement. ${ }^{20}$ We established that PI3K, but not cAMP, participates in M2 polarization by GA, and like a previous study of human monocytes, ${ }^{21}$ further supports a central role for PI3K/Akt in regulating inflammatory responses. These results also suggest that GA, or its peptide degradation products, might engage protein receptors. GA exhibits promiscuous binding to allelic surface MHC class II molecules. $^{22}$ Although the cytoplasmic domain of MHC class II molecules can participate in intracellular signaling, MHC class II is neither required for GA-dependent anti-inflammatory monocyte functions $^{3}$ nor necessary for targeting of GA to these cells in vivo. As data have indicated that GA can bind to CD11b (CD18), ${ }^{23,24}$ a cell surface $\alpha_{M} \beta_{2}$ integrin that participates in immune regulation, it was speculated that the interaction of GA with CD11b might contribute to GA-mediated modulation. However, we have observed that during in vivo GA treatment, GA or its byproducts accumulate equally well in CD11b-deficient monocytes as CD11b-bearing (WT) monocytes and that CD11b expression is not required for GA-mediated M2 differentiation (figure e-4). These findings highlight the challenges in identifying a specific receptor that binds GA and leads to activation of second messengers. Indeed, GA, a random linear arrangement of glutamate, lysine, alanine, and tyrosine (i.e., glat in glatiramer) that exhibits extensive sequence variation, may interact with many macromolecules.

Other random basic synthetic copolymers have also been considered for development as potential therapies in MS. ${ }^{25}$ In this regard, one random copolymer containing phenylalanine, lysine, alanine, and tyrosine and another composed of valine, lysine, 
alanine, and tryptophan also prevent EAE and cause immune modulation. ${ }^{25,26}$ Further, lysine-the basic amino acid common to these copolymers-is essential for therapeutic benefit, as "gat"-iramer acetate, lacking lysine, was ineffective in preclinical EAE studies. Thus, the observation that other synthetic random basic copolymers that, like GA, contain alanine, lysine, and an aromatic amino acid (i.e., phenylalanine, tryptophan, or tyrosine) also induce immune modulation raises the possibility that the signaling pathways modulated by GA identified in this report may be common to this class of copolymers.

While the observation that GA exerts antiinflammatory activity via inhibition of TRIF and its downstream signaling molecules is novel, it is known that the glucocorticoid dexamethasone also promotes M2 polarization. ${ }^{27}$ Although some data indicate that steroids interfere with NF-кB-dependent signaling, other results have demonstrated that steroid-mediated M2 polarization is primarily associated with inhibition of both IRF3 activation and type I IFN signaling. ${ }^{28}$ Resveratrol, an antioxidant found in red wine, inhibits TRIF-dependent activation, ${ }^{29}$ and, like GA, reduces clinical EAE and macrophage-mediated inflammatory activity. ${ }^{30}$ Thus, while the mechanism of action of GA is unique among MS therapeutics, suppression of TRIF-dependent signal transduction by agents that exhibit anti-inflammatory activity is not unprecedented.

Our finding that GA prevents IFN- $\beta$ synthesis in M2 monocytes is consistent with the earlier demonstration that STAT1 phosphorylation is reduced upon activation in these cells. ${ }^{3}$ One may find these observations surprising, considering that IFN- $\beta$ is approved for treatment of MS and has been protective in some EAE models. ${ }^{5,6}$ However, GA and IFN- $\beta$ promote immune modulation via distinct mechanisms. For example, GA and IFN- $\beta$ not only exhibit opposing activities on STAT1 phosphorylation, an obligate initial step in IFN- $\beta$ signaling, ${ }^{31}$ but also demonstrate distinct effects on proinflammatory T-cell differentiation. Whereas GA induces both Th2 and Treg cells that can modulate Th1 and Th17 responses, ${ }^{3,18}$ respectively, some data indicate that IFN- $\beta$ downregulates pathogenic Th1, but not Th17 responses. ${ }^{32}$ Separately, it was observed that deficiency in either IRF3 or JNK1 reduced susceptibility to EAE and impaired expansion of Th17 cells. ${ }^{9,33}$ Thus, our findings that GA treatment inhibited activation of JNK1 and nuclear translocation of IRF3 in monocytes and reduced production of IFN- $\beta$ are consistent both with the knowledge that inhibition of TRIF-dependent gene products reduces Th17 differentiation and that GA inhibits Th17 polarization. ${ }^{3}$ Together with the observation that GA prevented nuclear translocation of ATF-2, which contributes to the activation of the IFN- $\beta$ promoter, our data indicate that GA inhibits formation of the IFN- $\beta$ enhanceosome, a complex that is required for maximal IFN- $\beta$ gene transcription.

Considerable effort has been devoted to the identification of therapeutics that may provide additive or synergistic benefit when combined for treatment of MS. ${ }^{34-37}$ Even though mixed results were obtained when GA was tested in combination with type I IFNs in preclinical studies, ${ }^{38,39} \mathrm{GA}$ and intramuscular IFN$\beta$-1a were tested separately and together in a phase III clinical trial in relapsing-remitting MS. ${ }^{35}$ GA alone reduced relapse rate more than IFN- $\beta-1 \mathrm{a}$, and was equivalent to the combination of GA and IFN- $\beta$. While tempting to translate our mechanistic findings in relation to those clinical results, one should exercise caution; our study has focused primarily on identification of the mechanisms employed by GA in M2 monocyte polarization. Further, while results of that phase III MS trial did not identify clinical benefit from the combination of GA and intramuscular IFN- $\beta$-1a, it did not provide clear evidence of antagonism either. Evidence of synergy or antagonism may be dependent upon dosing. For example, when cholesterol-lowering statins were administered in combination with low-dose (intramuscular) or highdose (subcutaneous) IFN- $\beta$ in MS clinical trials, antagonism was observed and appeared more evident with high-dose IFN- $\beta^{40,41}$; this effect may relate to the opposing actions of these medications on STAT1 activation. ${ }^{36}$ In retrospect, our observation here that GA treatment inhibited expression of IFN- $\beta$ in monocytes would not have provided mechanistic support for testing the combination of GA and IFN- $\beta$ in MS. Recognizing that GA regulates TRIF-dependent IFN- $\beta$ production in monocytes may provide insight regarding its therapeutic effects in MS and for identification of surrogate markers of its efficacy.

\section{AUTHOR CONTRIBUTIONS}

N.M., T.P., and S.S.Z. designed research, analyzed data, and wrote the article. J.C.P., C.M.S., M.S.W., and P.H.L. analyzed data, gave conceptual advice, and discussed the results. N.M. and T.P. performed the experiments. U.S.-T. conducted the EAE experiments. S.S.Z. supervised the study. All authors read, commented, and approved the final manuscript.

\section{STUDY FUNDING}

Supported by grants to S.S.Z. from NIH (RO1 AI073737 and RO1 NS 063008), the National Multiple Sclerosis Society (RG 4124, RG 4786, and RG 5180), the Maisin Foundation, and Guthy Jackson Charitable Foundation; by a grant to P.H.L. from the Swiss National Science Foundation (310030-153164); N.M. is a recipient of an advanced researcher fellowship from the Swiss National Science Foundation \#PA00A-119532 and recipient of an advanced researcher exchange 2011 fellowship from the European Committee for Treatment and Research in Multiple Sclerosis (ECTRIMS) Foundation; T.P. was a recipient of postdoctoral fellowships from the NMSS and Teva Pharmaceutical Industries Ltd.

\section{DISCLOSURE}

N. Molnarfi reports no disclosures. T. Prod'homme is employed by Momenta Pharmaceuticals. U. Schulze-Topphoff is employed by Silence 
Therapeutics GmbH. C.M. Spencer reports no disclosures. M.S. Weber is an academic editor for PLoS One. J.C. Patarroyo is employed by Vedanta Biosciences. P.H. Lalive is on the scientific advisory board for Biogen-Idec and Novartis and received travel funding and/or speaker honoraria from Biogen-Idec, Teva, and Merck Serono. S.S. Zamvil served on the scientific advisory boards for BioMS, Teva Pharmaceuticals, Eli Lilly, and Myelin Repair Foundation; is deputy editor for Neurology: Neuroimmunology \& Neuroinflammation; has a patent pending for Aquaporin-4 peptides and methods for using the same; has consulted for Biogen Idec, Teva Neuroscience, EMD-Serono, Genzyme, Novartis, and Roche; is on the speakers' bureau for Advanced Health Media and Biogen Idec; and received research support from NIH, NMSS, and the Alexander M. and June L. Maisin Foundation. Go to Neurology.org/nn for full disclosure forms.

Received June 5, 2015. Accepted in final form September 29, 2015.

\section{REFERENCES}

1. Weber MS, Starck M, Wagenpfeil S, Meinl E, Hohlfeld R, Farina C. Multiple sclerosis: glatiramer acetate inhibits monocyte reactivity in vitro and in vivo. Brain 2004; 127:1370-1378.

2. Kim HJ, Ifergan I, Antel JP, et al. Type 2 monocyte and microglia differentiation mediated by glatiramer acetate therapy in patients with multiple sclerosis. J Immunol 2004;172:7144-7153.

3. Weber MS, Prod'homme T, Youssef S, et al. Type II monocytes modulate $\mathrm{T}$ cell-mediated central nervous system autoimmune disease. Nat Med 2007;13:935-943.

4. Prod'homme T, Zamvil SS. Bench to bedside: tempering antigen-presenting cells in multiple sclerosis. Nat Med 2008;14:614-615.

5. Prinz M, Schmidt H, Mildner A, et al. Distinct and nonredundant in vivo functions of IFNAR on myeloid cells limit autoimmunity in the central nervous system. Immunity 2008;28:675-686.

6. Guo B, Chang EY, Cheng G. The type I IFN induction pathway constrains Th17-mediated autoimmune inflammation in mice. J Clin Invest 2008;118:1680-1690.

7. Prinz M, Garbe F, Schmidt H, et al. Innate immunity mediated by TLR 9 modulates pathogenicity in an animal model of multiple sclerosis. J Clin Invest 2006;116:456-464.

8. Yamamoto M, Sato S, Hemmi H, et al. Role of adaptor TRIF in the MyD88-independent toll-like receptor signaling pathway. Science 2003;301:640-643.

9. Fitzgerald DC, O’Brien K, Young A, Fonseca-Kelly Z, Rostami A, Gran B. Interferon regulatory factor (IRF) 3 is critical for the development of experimental autoimmune encephalomyelitis. J Neuroinflammation 2014;11:130.

10. Burger D, Molnarfi N, Weber MS, et al. Glatiramer acetate increases IL-1 receptor antagonist but decreases $\mathrm{T}$ cellinduced IL-1beta in human monocytes and multiple sclerosis. Proc Natl Acad Sci USA 2009;106:4355-4359.

11. Molnarfi N, Gruaz L, Dayer JM, Burger D. Opposite regulation of IL-1beta and secreted IL-1 receptor antagonist production by phosphatidylinositide-3 kinases in human monocytes activated by lipopolysaccharides or contact with T cells. J Immunol 2007;178:446-454.

12. Kawai T, Adachi O, Ogawa T, Takeda K, Akira S. Unresponsiveness of MyD88-deficient mice to endotoxin. Immunity 1999;11:115-122.

13. Toshchakov V, Jones BW, Perera PY, et al. TLR4, but not TLR2, mediates IFN-beta-induced STAT1alpha/betadependent gene expression in macrophages. Nat Immunol 2002;3:392-398.
14. Panne D, Maniatis T, Harrison SC. An atomic model of the interferon-beta enhanceosome. Cell 2007;129:1111-1123.

15. Zhang B, Li M, Chen L, et al. The TAK1-JNK cascade is required for IRF3 function in the innate immune response. Cell Res 2009;19:412-428.

16. Ouwens DM, de Ruiter ND, van der Zon GC, et al. Growth factors can activate ATF2 via a two-step mechanism: phosphorylation of Thr71 through the Ras-MEKERK pathway and of Thr69 through RalGDS-Src-p38. EMBO J 2002;21:3782-3793.

17. Aharoni R, Teitelbaum D, Leitner O, Meshorer A, Sela M, Arnon R. Specific Th2 cells accumulate in the central nervous system of mice protected against experimental autoimmune encephalomyelitis by copolymer 1 . Proc Natl Acad Sci USA 2000;97:11472-11477.

18. Duda PW, Schmied MC, Cook SL, Krieger JI, Hafler DA. Glatiramer acetate (Copaxone) induces degenerate, Th2polarized immune responses in patients with multiple sclerosis. J Clin Invest 2000;105:967-976.

19. Prod'homme T, Weber MS, Steinman L, Zamvil SS. A neuropeptide in immune-mediated inflammation, Y? Trends Immunol 2006;27:164-167.

20. El Zein N, Badran B, Sariban E. VIP differentially activates beta2 integrins, CR1, and matrix metalloproteinase-9 in human monocytes through cAMP/PKA, EPAC, and PI$3 \mathrm{~K}$ signaling pathways via VIP receptor type 1 and FPRL1. J Leukoc Biol 2008;83:972-981.

21. Carpintero R, Brandt KJ, Gruaz L, Molnarfi N, Lalive PH, Burger D. Glatiramer acetate triggers PI3Kdelta/Akt and MEK/ERK pathways to induce IL-1 receptor antagonist in human monocytes. Proc Natl Acad Sci USA 2010;107:17692-17697.

22. Fridkis-Hareli M, Strominger JL. Promiscuous binding of synthetic copolymer 1 to purified HLA-DR molecules. J Immunol 1998;160:4386-4397.

23. Stapulionis R, Oliveira CL, Gjelstrup MC, et al. Structural insight into the function of myelin basic protein as a ligand for integrin alpha M beta 2. J Immunol 2008;180:3946-3956.

24. Toker A, Slaney CY, Backstrom BT, Harper JL. Glatiramer acetate treatment directly targets CD11b(+)Ly6G(-) monocytes and enhances the suppression of autoreactive $\mathrm{T}$ cells in experimental autoimmune encephalomyelitis. Scand J Immunol 2011;74:235-243.

25. Fridkis-Hareli M, Santambrogio L, Stern JN, Fugger L, Brosnan C, Strominger JL. Novel synthetic amino acid copolymers that inhibit autoantigen-specific $\mathrm{T}$ cell responses and suppress experimental autoimmune encephalomyelitis. J Clin Invest 2002;109:1635-1643.

26. Stern JN, Illes Z, Reddy J, et al. Amelioration of proteolipid protein 139-151-induced encephalomyelitis in SJL mice by modified amino acid copolymers and their mechanisms. Proc Natl Acad Sci USA 2004;101:11743-11748.

27. Martinez FO, Gordon S. The M1 and M2 paradigm of macrophage activation: time for reassessment. F1000prime Rep 2014;6:13.

28. Reily MM, Pantoja C, Hu X, Chinenov Y, Rogatsky I. The GRIP1:IRF3 interaction as a target for glucocorticoid receptor-mediated immunosuppression. EMBO J 2006; 25:108-117.

29. Youn HS, Lee JY, Fitzgerald KA, Young HA, Akira S, Hwang DH. Specific inhibition of MyD88-independent signaling pathways of TLR3 and TLR4 by resveratrol: molecular targets are TBK1 and RIP1 in TRIF complex. J Immunol 2005;175:3339-3346. 
30. Imler TJ Jr, Petro TM. Decreased severity of experimenta autoimmune encephalomyelitis during resveratrol administration is associated with increased IL-17+IL-10+ T cells, CD4(-) IFN-gamma+ cells, and decreased macrophage IL-6 expression. Int Immunopharmacol 2009;9:134-143.

31. Darnell JE, Kerr IM, Stark GR. Jak-STAT pathways and transcriptional activation in response to IFNs and other extracellular signaling proteins. Science 1994;264: 1415-1421.

32. Axtell RC, de Jong BA, Boniface $\mathrm{K}$, et al. T helper type 1 and 17 cells determine efficacy of interferon-beta in multiple sclerosis and experimental encephalomyelitis. Nat Med 2010;16:406-412.

33. Tran EH, Azuma YT, Chen M, Weston C, Davis RJ, Flavell RA. Inactivation of JNK1 enhances innate IL-10 production and dampens autoimmune inflammation in the brain. Proc Natl Acad Sci USA 2006;103: 13451-13456.

34. Stuve O, Youssef S, Weber MS, et al. Immunomodulatory synergy by combination of atorvastatin and glatiramer acetate in treatment of CNS autoimmunity. J Clin Invest 2006;116:1037-1044.

35. Lublin FD, Cofield SS, Cutter GR, et al. Randomized study combining interferon and glatiramer acetate in multiple sclerosis. Ann Neurol 2013;73:327-340.
36. Zamvil SS, Steinman L. Combining statins with interferon beta in multiple sclerosis: think twice, it might not be all right. Lancet Neurol 2011;10:672-673.

37. Metz LM, Li D, Traboulsee A, et al. Glatiramer acetate in combination with minocycline in patients with relapsingremitting multiple sclerosis: results of a Canadian, multicenter, double-blind, placebo-controlled trial. Mult Scler 2009; 15:1183-1194.

38. Brod SA, Lindsey JW, Wolinsky JS. Combination therapy with glatiramer acetate (copolymer-1) and a type I interferon (IFN-alpha) does not improve experimental autoimmune encephalomyelitis. Ann Neurol 2000;47:127-131.

39. Soos JM, Stuve O, Youssef S, et al. Cutting edge: oral type I IFN-tau promotes a Th2 bias and enhances suppression of autoimmune encephalomyelitis by oral glatiramer acetate. J Immunol 2002;169:2231-2235.

40. Birnbaum G, Cree B, Altafullah I, Zinser M, Reder AT. Combining beta interferon and atorvastatin may increase disease activity in multiple sclerosis. Neurology 2008;71: 1390-1395.

41. Sorensen PS, Lycke J, Eralinna JP, et al. Simvastatin as add-on therapy to interferon beta-1a for relapsingremitting multiple sclerosis (SIMCOMBIN study): a placebo-controlled randomised phase 4 trial. Lancet Neurol 2011;10:691-701. 


\section{Neurology \\ Neuroimmunology \& Neuroinflammation}

\section{Glatiramer acetate treatment negatively regulates type $I$ interferon signaling Nicolas Molnarfi, Thomas Prod'homme, Ulf Schulze-Topphoff, et al. \\ Neurol Neuroimmunol Neuroinflamm 2015;2; \\ DOI 10.1212/NXI.0000000000000179}

This information is current as of November 9, 2015

\section{Updated Information \& Services \\ Supplementary Material \\ References \\ Subspecialty Collections}

Errata

Permissions \& Licensing

Reprints including high resolution figures, can be found at: http://nn.neurology.org/content/2/6/e179.full.html

Supplementary material can be found at: http://nn.neurology.org/content/suppl/2015/12/04/2.6.e179.DC1

This article cites 41 articles, 15 of which you can access for free at: http://nn.neurology.org/content/2/6/e179.full.html\#\#ref-list-1

This article, along with others on similar topics, appears in the following collection(s):

Autoimmune diseases

http://nn.neurology.org//cgi/collection/autoimmune_diseases Multiple sclerosis

http://nn.neurology.org//cgi/collection/multiple_sclerosis

An erratum has been published regarding this article. Please see next page or:

/content/2/6/e189.full.pdf

Information about reproducing this article in parts (figures,tables) or in its entirety can be found online at:

http://nn.neurology.org/misc/about.xhtml\#permissions

Information about ordering reprints can be found online: http://nn.neurology.org/misc/addir.xhtml\#reprintsus

Neurol Neuroimmunol Neuroinflamm is an official journal of the American Academy of Neurology.

Published since April 2014, it is an open-access, online-only, continuous publication journal. Copyright $\odot$ 2015 American Academy of Neurology. All rights reserved. Online ISSN: 2332-7812.

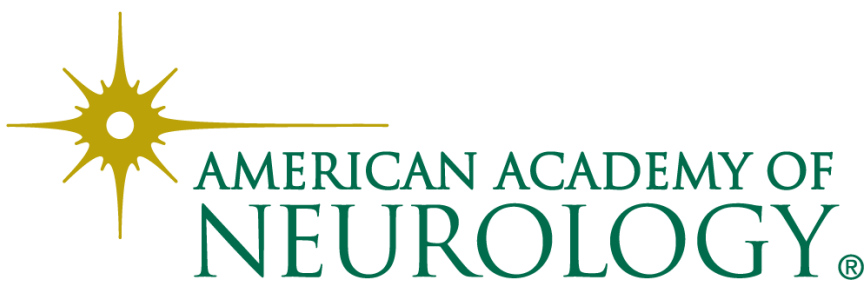




\section{CORRECTION}

\section{Glatiramer acetate treatment negatively regulates type I interferon signaling}

In the article "Glatiramer acetate treatment negatively regulates type I interferon signaling" by N. Molnarfi et al. (Neurol Neuroimmunol Neuroinflamm 2015;2:e179. DOI: 10.1212/NXI.0000000000000179), originally published on November 9, 2015, there is an error in the description of figure 2B. This figure shows IкB $\alpha$ degradation, not IкB $\alpha$ phosphorylation as originally described. In Methods ("Western blot"), the second sentence should read "Nitrocellulose membranes were probed $\left(4^{\circ} \mathrm{C}\right.$, overnight) with antibodies against phospho-Akt1 (Ser473), phospho-JNK (Thr183/ Tyr185), phospho-p38 MAP kinase (Thr180/Tyr182), IкBa, glyceraldehyde-3-phosphate dehydrogenase (GAPDH), total STAT1, and total Akt1/2/3 (Cell Signaling Technology, Danvers, MA)." In figure 2, the label above panel B should read just "ІкВа" and the corresponding sentence in the legend should read "... and membranes were probed for IкBa." In the second paragraph under Results ("GA inhibits TRIF-dependent, but not MyD88-dependent, signaling"), the 13th sentence should read "As shown in figure 2B, GA exposure did not impair degradation of the inhibitor protein IKBa following LPS treatment.” The authors regret this error. 